\title{
Telomeric repeat factor 1 protein levels correlates with telomere length in colorectal cancer
}

\author{
Cristina Valls-Bautista ${ }^{1,2}$, Carme Piñol-Felis ${ }^{1,2}$, Josep M. Reñé-Espinet ${ }^{1,2,3}$, Juan Buenestado-García ${ }^{1,2,3}$ \\ and Joan Viñas-Salas ${ }^{2,3,4}$ \\ ${ }^{1}$ Department of Medicine. School of Medicine. University of Lleida, Spain. ${ }^{2}$ IRBLleida. Spain. ${ }^{3}$ Hospital Universitari \\ Arnau de Vilanova. Lleida, Spain. ${ }^{4}$ Department of Surgery. School of Medicine. University of Lleida. Lleida, Spain
}

\begin{abstract}
Background: colorectal cancer is the third cancer cause of death in Spain. It is important to investigate new tumoral markers for early diagnosis, disease monitoring and prevention strategies. Telomeres protect the chromosome from degradation by nucleases and endto-end fusion. The progressive loss of the telomeric ends of chromosomes is an important mechanism in the timing of human cellular aging. Telomeric Repeat Factor 1 (TRF1) is a protein that binds at telomere ends.

Purpose: to measure the concentrations of TRF1 and the relationships among telomere length, telomerase activity, and TRF1 levels in tumor and normal colorectal mucosa.

Method: from normal and tumoral samples of 83 patients who underwent surgery for colorectal cancer we analyzed TRF1 protein concentration by Western Blot, telomerase activity, by the fluorescent-telomeric repeat amplification protocol assay and telomere length by Southern Blot.

Results: high levels of TRF1 were observed in $68.7 \%$ of tumor samples, while the majority of normal samples (59\%) showed negative or weak TRF1 concentrations. Among the tumor samples, telomere length was significantly associated with TRF1 protein levels $(p=0.023)$.

Conclusions: a relationship was found between telomere length and TRF1 abundance protein in tumor samples, which means that TRF1 is an important factor in the tumor progression and maybe a diagnostic factor.
\end{abstract}

Key words: Telomeric repeat factor 1 . Telomere length. Telomerase activity. Colorectal cancer.

Valls-Bautista C, Piñol-Felis C, Reñé-Espinet JM, BuenestadoGarcía J, Viñas-Salas J. Telomeric repeat factor 1 protein levels correlates with telomere length in colorectal cancer. Rev Esp Enferm Dig 2012; 104:530-536.

Received: 25-06-2012

Accepted: 25-09-2012

Correspondence: Carme Piñol-Felis. School of Medicine. Hospital Universitari Arnau de Vilanova. Rovira Roure, 80. 25198 Lleida, Spain e-mail: pinyol@medicina.udl.cat

\author{
ABBREVIATIONS \\ ALT: alternative lengthening of telomeres. \\ $\mathrm{Kb}$ : kilobases. \\ TA: telomerase activity. \\ TI: telomerase index. \\ TL: telomere length. \\ TPG: total product generated. \\ TRF1: telomeric repeat factor 1. \\ TRF2: telomeric repeat binding factor 2 .
}

\section{INTRODUCTION}

Telomeres, structures composed mostly of chromatin localized at the end of eukaryotic chromosomes (1), consist of 800-3000 tandem repeats of six nucleotides (' 'TTAGGG3') along a 5-15 kilobases ( $\mathrm{Kb}$ ) sequence (2). Telomeres protect the chromosome from degradation by nucleases and end-toend fusion. The progressive loss of the telomeric ends of chromosomes is an important mechanism in the timing of human cellular aging (3). When telomeres become sufficiently short, the majority of cells enter in senescence, but some escape because they are able to maintain their telomere length (TL).

Telomerase is a ribonucleoprotein that compensates for the shortening of telomeres by adding TTAGGG repeats onto the chromosome ends (4). In germ cells and renewing tissues, telomerase is activated and maintains the integrity and stability of the genome (5). Some immortalized mammalian cell lines and tumors maintain or increase the overall length of their telomeres in the absence of telomerase activity (TA) by one or more mechanisms referred to as alternative lengthening of telomeres (ALT) (6).

Previous studies have not uncovered a direct relationship between TA and TL (7), raising the possibility of telomerebinding proteins that regulate TL, TA, and maintain the protective structure of the telomere in vivo $(8,9)$. Six proteins (TRF1, TRF2, POT1, TIN2, TPP1, and hRap1) bind at telom- 
ere ends in a complex known as shelterin. TRF1, TRF2, and POT1 directly bind telomeric DNA. Telomeric Repeat Binding Factor 1 (TRF1) acts in cis, obstructing telomerase and preventing telomere elongation (10). The number of molecules of TRF1 bound to each telomere end is correlated with TL (11). Telomeric Repeat Binding Factor 2 (TRF2) induces Tloop structure, protecting telomeres from degradation by exonucleases. The telomere homeostasis hypothesis predicts that longer telomeres bind more TRF1; TRF1 obstructs telomerase and prevents telomere elongation (12). Short telomeres bind fewer TRF1 molecules, favoring an open conformation with more chances to be elongated by telomerase (13).

TL abnormalities appear to be one of the earliest prevalent genetic alterations acquired during the multistep process of malignant transformation (14). By demonstrating the participation of TRF1 in TL maintenance, we gain a useful new biomarker to employ in early diagnostics and disease monitoring and prevention strategies.

In this study, we investigate whether the telomere homeostasis hypothesis can be directly applied to understand various molecular characteristics of normal and cancerous tissues. We measured TA, TL, and TRF1 protein abundance in cancerous and noncancerous mucosal samples from 83 colorectal cancer patients. Finally, we applied statistical techniques to determine the relationships among these three variables and additional anatomopathological features.

\section{PATIENTS AND METHODS}

\section{Patients}

Fresh samples of cancer tissue and normal mucosa were obtained from 83 patients (46 male, 37 female) undergoing oncological tumor resection for colorectal cancer at Lleida Arnau de Vilanova University Hospital. We considered normal mucosa as having a macroscopically normal appearance and lying from $10 \mathrm{~cm}$ to the tumor. The patients ranged in age from 45 to 89 years, with an average age of 69.3 years. No patient's received neoadjuvant therapy. All tissue samples weighing $100 \mathrm{mg}$ were immediately frozen in liquid nitrogen and stored at $-80{ }^{\circ} \mathrm{C}$.

Samples were analyzed by the hospital's Pathology unit. Tumors were staged according to the tumor-node-metastases classification of the International Union against Cancer (UICC-TNM classification). Clinical and pathological data were collected in a specific database. All research protocols used in this study were approved by the Hospital Clinical Research Ethics Committee, conforms Ethical Guidelines of Helsinki Declaration.

\section{Evaluation of TRF1 protein concentration by Western Blot}

Briefly, $40 \mathrm{mg}$ of frozen tissue samples were homogenized with $400 \mu \mathrm{l}$ protein lysis buffer (125 mM Tris,
pH 6.8), with RNase inhibitor (RNAsin® Ribonuclease inhibitor, Promega) added to the tumor samples. The homogenates were centrifuged at $12,000 \mathrm{rpm}$ at $4{ }^{\circ} \mathrm{C}$ for 2 minutes, and the supernatants were sonicated (Digital sonifier 450 Branson) to degrade DNA. Total protein concentration was determined using the Bio-Rad Micro Protein Assay (Bio-Rad Laboratories). $25 \mu \mathrm{g}$ of total protein were separated by electrophoresis on $10 \%$ agarose, transferred to a PDVF membranes (polyvinylidine fluoride, Millipore) and incubated with 1:500 anti-TRF1 monoclonal mouse antibody into a 5\% milk solution (clone TRF-78, SigmaAldrich) under the manufacturer's recommended conditions. The corresponding peroxidase-labeled secondary anti-mouse antibody, diluted 1:12,500 (Jackson ImmunoResearch Laboratories), was detected using an enhanced chemiluminescence system (EZ-ECL Chemiluminescence Detection Kit for HRP, Biological Industries Kibbutz Beit Haemek, Israel) and analyzed by Lumi-Imager (Boehringer Mannheim). Levels of monoclonal anti- $\alpha$-tubulin (Sigma, Sant Louis, Missouri) used at 1/30,000 dilution were measured for protein normalization between samples. TRF1 levels were qualitatively compared with a TRF1 positive control (cell line 293, Human embryonic kidney cells) (15).

Levels of TRF1 protein in tumor and normal samples were determined relative to protein levels in cell line 293, our baseline positive control (designated "100\%"). Samples were categorized by three separate observers as follows: "-" as low concentration relative to the positive control, "+" as weak concentration, "++" as medium concentration, and " +++ " as high concentration. We further sub-classified TRF1 protein levels into two groups: samples with low and weak concentrations (-/+) and samples with medium and high concentrations $(++/+++)$. To determine the TRF1 ratio we compared the protein levels in two samples from the same patient. The ratio was positive if the TRF1 level was higher in the tumor mucosa than the normal mucosa, and the ratio was negative if the TRF1 level was lower in the tumor mucosa than the normal mucosa. These TRF1 ratios were divided into two groups: negative and equal ratios (/=), and positive ratios $(+)$.

\section{Fluorescent-telomeric repeat amplification protocol}

TA was measured quantitatively by the fluorescent-telomeric repeat amplification protocol assay (TRAP-F), using a TRAPeze Telomerase Detection Kit (INTERGEN ${ }^{\circledR}$ Purchase) (16). We report TA in TPG (total product generated). Analysis was performed as described previously (17). Telomerase index (TI) was determined by the formula $\mathrm{TI}=\log$ (TA of cancer tissue-TA of normal mucosa).

\section{Telomere length measurement by Southern Blot}

Telomere restriction fragment (TRF) length was determined by Southern blot (Telo TAGGG Telomere length 

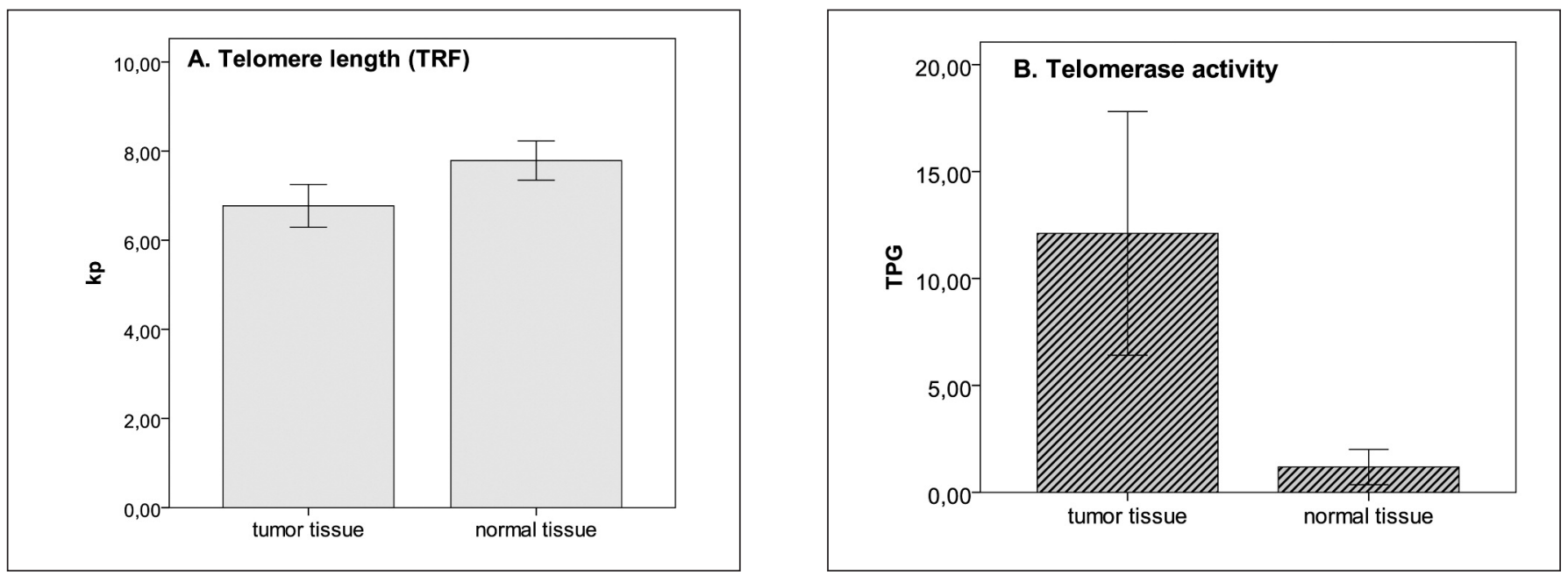

Fig. 1. Telomere length and telomerase activity in tumor and normal tissue in colorectal cancer patients. A. Telomere length levels in kb. B. Telomerase activity in TPG in tumor and normal tissue of colorectal cancer patients $(n=83)$. Data are shown as mean \pm standard error. In both $p<0.001$.

assay, Roche Diagnostics) in tumor and normal tissues. Analysis was performed as described previously $(18,19)$. The telomere length was expressed in $\mathrm{Kb}$. The telomere length ratio (TLR) was determined as the quotient between the TL in the tumor tissue and the TL in normal mucosa from the same patient. Telomere shortening and elongation were defined as TLs of carcinoma less than $80 \%$ and greater than $120 \%$ of the corresponding normal mucosa, respectively.

\section{Statistical analysis}

We used the statistical program SPSS ${ }^{\circledR} 18.0$ for Windows (SPSS Inc.) for analyses. Patient factors and TRF1 expression level were analyzed using the chi-square test. We used the non-parametric Mann-Whitney U-test and Fisher test. The cut-off TRF length ratio $=1$ was chosen by means of the classification and regression tree technique (CART). A TI value of 0.85 was used to classify patients showing a high index (>0.85) and patients with low index ("0.85)

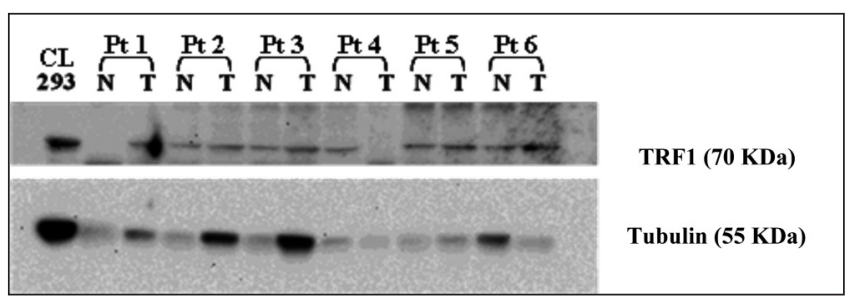

Fig. 2. TRF1 analysis by Western Blot. A representative Western Blot analysis is shown for telomeric repeat binding factor 1 (TRF1) expression in patients $(\mathrm{Pt})$ with colorectal carcinoma. Results obtained from five tumor tissues $(\mathrm{T})$ and non-tumor tissues $(\mathrm{N})$ are included, and tubulin normalization also is indicated. First line is a TRF1 positive control (cell line 293, Human embryonic kidney cells). and this value was estimated using maximally selected chisquared statistics (18). We considered results statistically significant when $p<0.05$.

\section{RESULTS}

\section{Molecular markers differ between cancerous and normal tissues}

The anatomopathological characteristics studied from 83 colorectal cancer patients are shown in table I. Mean TL (Fig. 1A) in tumor samples was $6.77 \mathrm{~kb}(2.19-13.31 \mathrm{~kb})$, while in normal mucosa the average TL was $7.78 \mathrm{~kb}(4.20-$ $15.70 \mathrm{~kb})(p<0.001)$. The mean TA (Fig. 1B) in the tumor samples was 12.3 TPG (0-164.87 TPG) and in normal samples it was 1.18 TPG $(0-24.67$ TPG) $(p<0.001)$. The TI mean was 0.45 . Additionally, we detected TRF1 protein in $95 \%$ and $89 \%$ of our cancerous and noncancerous samples, respectively. The majority of normal samples $(59 \%)$ showed negative or weak TRF1 concentration, while $68.7 \%$ of tumor samples presented high levels of TRF1. To summarize, on average the cancerous samples had shorter telomeres, higher TA, and higher TRF1 protein levels than paired noncancerous samples.

\section{TRF1 protein levels are significantly related to anatomopathological features (Fig. 2)}

Two statistically significant relationships between the anatomopathological variables and molecular tissue markers emerged upon chi-square analysis (Tables I and II). First, normal mucosa from male patients exhibited lower relative TRF1 protein levels compared with the noncancerous samples from female patients (Table I, $p<0.009$ ). 
Table I. Relationships among TRF1 protein levels (low/weak or medium/high) and anatomopathological features analyzed by the Chi-square test

\begin{tabular}{|c|c|c|c|c|c|c|}
\hline \multirow[t]{2}{*}{ Variable (n) } & \multicolumn{2}{|c|}{ TRF1 level, tumor (\%) } & \multicolumn{2}{|c|}{ TRF1 level, normal (\%) } & \multicolumn{2}{|c|}{ TRF1 Ratio (\%) } \\
\hline & $-/+$ & $++/+++$ & $-/+$ & $++/+++$ & $-/=$ & + \\
\hline Patients $(n=83)$ & $26(31)$ & $57(69)$ & $49(59)$ & $34(41)$ & $49(59)$ & $34(41)$ \\
\hline \multicolumn{7}{|l|}{ Gender } \\
\hline Male (46) & $18(39)$ & $28(61)$ & $33(72)$ & $13(28)$ & $20(43.5)$ & $26(56.5)$ \\
\hline Female (37) & $8(22)$ & $29(78)$ & $16(43)$ & $21(57)$ & $17(46)$ & $20(54)$ \\
\hline$p$ & 0.087 & & 0.009 & & 0.822 & \\
\hline \multicolumn{7}{|l|}{ Age (years) } \\
\hline " $70(41)$ & $9(22)$ & $32(78)$ & $25(61)$ & $16(39)$ & $15(37)$ & $26(63)$ \\
\hline$>70(42)$ & $17(40.5)$ & $25(59.5)$ & $24(57)$ & $18(43)$ & $22(52)$ & $20(48)$ \\
\hline$p$ & 0.069 & & 0.723 & & 0.148 & \\
\hline \multicolumn{7}{|l|}{ Tumor site } \\
\hline Right colon (32) & $10(31)$ & $22(69)$ & $17(53)$ & $15(47)$ & $17(53)$ & $15(47)$ \\
\hline Left colon (21) & $4(19)$ & $17(81)$ & $15(71)$ & $6(29)$ & $5(24)$ & $16(76)$ \\
\hline Rectum (30) & $12(40)$ & $18(60)$ & $17(57)$ & $13(43)$ & $15(50)$ & $15(50)$ \\
\hline$p$ & 0.284 & & 0.393 & & 0.083 & \\
\hline \multicolumn{7}{|l|}{ Tumor histology } \\
\hline Adenocarcinoma (73) & $23(31.5)$ & $50(68.5)$ & $45(62)$ & $28(38)$ & $31(42.5)$ & $42(57.5)$ \\
\hline Mucinous (10) & $3(30)$ & $7(70)$ & $4(40)$ & $6(60)$ & $6(60)$ & $4(40)$ \\
\hline$p$ & 0.923 & & 0.192 & & 0.295 & \\
\hline \multicolumn{7}{|l|}{ Macroscopic aspect } \\
\hline Ulcerous-infiltrative (42) & $15(36)$ & $27(64)$ & $22(52)$ & $20(48)$ & $23(55)$ & $19(45)$ \\
\hline Vegetating (36) & $10(28)$ & $26(72)$ & $25(70)$ & $11(30)$ & $12(33)$ & $24(67)$ \\
\hline Polypoid (5) & $1(20)$ & $4(80)$ & $2(40)$ & $3(60)$ & $2(40)$ & $3(60)$ \\
\hline$p$ & 0.642 & & 0.209 & & 0.161 & \\
\hline \multicolumn{7}{|l|}{ Dukes classification } \\
\hline$A(1)$ & $1(100)$ & $0(0)$ & $1(100)$ & $1(0)$ & $1(100)$ & $0(0)$ \\
\hline$B(47)$ & $16(34)$ & $31(66)$ & $26(55)$ & $21(45)$ & $24(51)$ & $23(49)$ \\
\hline$C(24)$ & $6(25)$ & $18(75)$ & $16(67)$ & $8(33)$ & $10(42)$ & $14(58)$ \\
\hline $\mathrm{D}(11)$ & $3(27)$ & $8(73)$ & $6(54.5)$ & $5(45.5)$ & $2(18)$ & $9(82)$ \\
\hline$p$ & 0.410 & & 0.652 & & 0.156 & \\
\hline \multicolumn{7}{|l|}{$T$ classification } \\
\hline Tis $(2)$ & $1(50)$ & $1(50)$ & $2(100)$ & $0(0)$ & $1(50)$ & $1(50)$ \\
\hline $\mathrm{T} 2(11)$ & $3(27)$ & $8(73)$ & $5(45.5)$ & $6(54.5)$ & $6(54.5)$ & $5(45.5)$ \\
\hline T3 (63) & $20(32)$ & $43(68)$ & $35(56)$ & $28(44)$ & $29(46)$ & $34(54)$ \\
\hline $\mathrm{T} 4(7)$ & $2(29)$ & $5(71)$ & $7(100)$ & $0(0)$ & $1(14)$ & $6(86)$ \\
\hline$p$ & 0.932 & & 0.060 & & 0.373 & \\
\hline \multicolumn{7}{|l|}{ N classification } \\
\hline$N(-)(49)$ & $17(35)$ & $32(65)$ & $27(55)$ & $22(45)$ & $25(51)$ & $24(49)$ \\
\hline$N(+)(34)$ & $9(26.5)$ & $25(73.5)$ & $22(65)$ & $12(35)$ & $12(35)$ & $22(65)$ \\
\hline$p$ & 0.427 & & 0.382 & & 0.156 & \\
\hline \multicolumn{7}{|l|}{ M classification } \\
\hline $\mathrm{MO}(72)$ & $23(32)$ & $49(68)$ & $43(60)$ & $29(40)$ & $35(49)$ & $37(51)$ \\
\hline M1 (11) & $3(27)$ & $8(73)$ & $6(54.5)$ & $5(45.5)$ & $2(18)$ & $9(82)$ \\
\hline$p$ & 0.756 & & 0.745 & & 0.059 & \\
\hline \multicolumn{7}{|l|}{ TNM stage } \\
\hline $0(2)$ & $1(50)$ & $1(50)$ & $2(100)$ & $0(0)$ & $1(50)$ & $1(50)$ \\
\hline I (11) & $3(27)$ & $8(73)$ & $5(45.5)$ & $6(54.5)$ & $6(54.5)$ & $4(45.5)$ \\
\hline II (35) & $13(37)$ & $22(63)$ & $20(57)$ & $15(43)$ & $18(51)$ & $17(49)$ \\
\hline III (24) & $6(25)$ & $18(75)$ & $16(67)$ & $8(33)$ & $10(42)$ & $14(58)$ \\
\hline IV (11) & $3(27)$ & $8(73)$ & $6(54.5)$ & $5(45.5)$ & $2(18)$ & $9(82)$ \\
\hline$p$ & 0.829 & & 0.567 & & 0.365 & \\
\hline
\end{tabular}


Table II. Relationships among TRF1 protein levels (low/weak or medium/high) and telomere length, telomere length ratio and telomerase activity analyzed by the Chi-square test

\begin{tabular}{|c|c|c|c|c|c|c|}
\hline \multirow[t]{2}{*}{ Variable (n) } & \multicolumn{2}{|c|}{ TRF1 level, tumor (\%) } & \multicolumn{2}{|c|}{ TRF1 level, normal (\%) } & \multicolumn{2}{|c|}{ TRF1 Ratio (\%) } \\
\hline & $-/+$ & $++/+++$ & $-/+$ & $++/+++$ & $-l=$ & + \\
\hline Patients $(\mathrm{n}=83)$ & $26(31)$ & $57(69)$ & $49(59)$ & $34(41)$ & $49(59)$ & $34(41)$ \\
\hline \multicolumn{7}{|l|}{ Telomere length } \\
\hline Shortened (32) & $15(47$ & $17(53)$ & $17(53)$ & $15(47)$ & $17(53)$ & $15(47)$ \\
\hline Elongated (7) & $3(43)$ & $4(57)$ & $4(57)$ & $3(47)$ & $4(57)$ & $3(43)$ \\
\hline No change (44) & $8(18)$ & $36(82)$ & $28(64)$ & $16(36)$ & $16(36)$ & $28(64)$ \\
\hline$p$ & 0.023 & & 0.651 & & 0.273 & \\
\hline \multicolumn{7}{|l|}{$T L R$} \\
\hline$" 1(63)$ & $21(33)$ & $42(67)$ & $38(60)$ & $25(40)$ & $26(41)$ & $37(59)$ \\
\hline$>1(20)$ & $5(25)$ & $15(75)$ & $11(55)$ & $9(45)$ & $11(55)$ & $9(45)$ \\
\hline$p$ & 0.484 & & 0.674 & & 0.282 & \\
\hline \multicolumn{7}{|l|}{ TI 0.85} \\
\hline " 0.85 (57) & $17(30)$ & $40(70)$ & $31(54)$ & $26(46)$ & $27(47)$ & $30(53)$ \\
\hline$>0.85(26)$ & $9(35)$ & $17(65)$ & $18(69)$ & $8(31)$ & $10(38.5)$ & $16(61.5)$ \\
\hline$p$ & 0.662 & & 0.202 & & 0.449 & \\
\hline
\end{tabular}

TLR: telomere lenght ratio; TI: telomerase index.

Second, we found among the tumor samples, TL had a significant association with TRF1 protein levels ( $p<$ $0.023)$; this association was not significant in the normal mucosal samples (Table II, $p<0.651$ ) the tumors that did not change their TL had a high TRF1 expression than the others that elongated or shorten their TL.

\section{TRF1 protein abundance, telomerase activity, and telomere length are related}

We uncovered a relationship between TA (positive/negative) and TRF1 protein abundance in tumor samples: the $87.5 \%$ of tumors samples with negative TA showed medium/high TRF1 protein levels, as compared with $64 \%$ of positive telomere tumors. This result was not statically significant $(p<0.071)$. Tumors with high TRF1 protein abundance exhibited greater TA and longer telomeres than tumors with lower TRF1 protein levels. In noncancerous samples, however, the samples with low TRF1 protein levels displayed higher TA and shorter telomeres than the noncancerous samples with high TRF1 protein abundance, a difference that was not statistically significant $(p<0.052)$.

\section{DISCUSSION}

In this study we have shown on average that cancerous samples had shorter telomeres, higher TA, and higher TRF1 protein levels than paired noncancerous samples. While on average tumoral mucosa display elevated TA and shorter telomeres relative to normal mucosa, no statistically significant association between TA and TL has been identified $(8,17,20)$. We identified TRF1 as a candidate protein for maintenance of telomere homeostasis. This protein, located at the end of telomeres, maintains the T-loop structure necessary to protect chromosome ends from degradation $(12,21)$.

TRF1 has been subjected to various studies in various tumor types $(13,14,21,22)$. The main methodologies to study TRF1 have been western blotting (13) and immunohistochemistry $(22,23)$. We detected TRF1 protein in $95 \%$ and $89 \%$ of our cancerous and noncancerous samples, respectively. The majority of normal samples showed low or weak TRF1 protein levels, while almost $70 \%$ of tumor samples presented high TRF1 protein abundance. This is an unexpected result; although we anticipated TRF1 protein in both tissues, we did not expect to observe elevated TRF1 levels in tumor samples relative to normal samples, since telomeres in tumor samples are shorter than normal mucosa and TRF1 protein was previously observed bound to the (longer) telomeres of normal mucosa $(10,13)$. Given these previous studies, we expected a direct association between TL and levels of TRF1. This association was not identified in tumor samples due to altered telomere homeostasis mechanisms. Generally, in normal mucosa in people without a tumoral process, this mucosa show a relation between TL and TRF1 levels, since longer telomeres accept more TRF1 bound because this binding is sequence specific. In tumoral mucosa the telomere homeostasis was deregulated, lots of cellular mechanisms not function well (due to a transformation process from normal to tumor cell). In addition, shorter telomeres may be more difficult to stabilizer and need to bind higher levels of TRF1. Whereas, in normal mucosa which has longer telomeres they may be easier to stabilizer and other mechanisms which control the homeostasis of telomeres function well and needs less TRF1 to bind.

TRF1 protein abundance in normal mucosa showed a significant association with gender, with more samples from women displaying elevated protein levels. High quantity of TRF1 upon normal mucosa means more stability for telom- 
eres and homeostasis of them. This situation determines healthier telomeric homeostasis in females than in males. Several studies reported that females have long telomeres than males due to faster attrition rate in males (in people without tumoral process) (24). Nawrot et al. (25) concluded that the difference in gender were due to estrogen-responsive hormone regulators in telomerase. Nawrot suggested that the process of ageing might be an X-linked trait. TL had a significant association with TRF1 protein levels in tumor mucosa: a high percentage of samples with average normal TL displayed high TRF1 protein levels. The binding of TRF1 on the telomeres may be a protection mechanism, as it can permit a better tolerance to shorter telomeres during tumor progression (26). TRF1 and TRF2 bind to the double-strand telomere DNA together with POT1, Rap1, TIN1, and PPT to form the shelterin complex, protecting chromosome ends and activating the telomere repair pathway (10). Under normal conditions, a direct relation exists between TRF1 levels and TL (12), but we have uncovered an indirect relation between TRF1 levels and TL in tumor samples. If telomeres in tumor samples have sufficient levels of bound TRF1, they may survive despite the short telomeres. TL and TA are altered in tumors (13); this study provides evidence that TRF1 levels are also altered in comparison to normal tissue. Previously, the percentage of TRF1-positive cells was correlated positively with tumor histology; differentiated tumors expressed more TRF1 -more differentiated level more TRF1 level (22). A significant negative relationship between TRF1 levels and histology degree was observed in brain tumors $(27,28)$.

While we observed that, as a group, telomerase-negative tumors displayed higher TRF1 protein levels than normal tissue; this result did not pass our significance cutoff. Perhaps tumors with low TA require additional mechanisms to maintain TL; for example, many TRF1 molecules binding at the telomeres may increase survival, as suggested by the homeostasis telomere hypothesis (6). It is now known that TRF1 binds the telomeres lengthwise, hindering telomerase binding and lengthening the telomeres $(10,11)$. Our results agree with a previous work demonstrating that longer telomeres recruited more TRF1 than shorter telomeres, and that TRF1 inhibited telomerase; in other words, telomerase tended to elongate shorter telomeres with less bound TRF1 (10). TRF1 seems to protect short telomeres short, maintaining their integrity and promoting tumor survival. Samples with medium to high TRF1 protein abundance showed longer telomeres than samples with low TRF1 expression in both normal and tumor tissues. These results agree with other studies affirming that the amount of TRF1 binding at the telomere end is proportional to the TL (12).

The statistical analyses did not reveal significant differences TA, TL, and TRF1 protein abundance. On the one hand, TL demonstrated a link with TRF1 protein abundance; on the other, TRF1 protein levels had no significant relation with TA. In general, we found that tumors with low TA had long telomeres and expressed high levels of TRF1, whereas tumors with higher TA had shorter telomeres and expressed lower TRF1 levels. A possible explanation for this behavior is for samples with low or no telomerase activity, a high level of TRF1 could become essential for chromosome stability (11), thus affecting TA. Additionally, the telomeres in tumor cells may display different behavior from telomeres in normal cells, possibly as a result of protein regulation of TL and TA (8). It is known that three sequence-specific DNA binding proteins (TRF1, TRF2, and POT1) are recruited to chromosomal ends; they form a functional complex that caps telomere ends and prevents telomere dysfunction which initiates genomic changes and promotes the development of cancer $(8,14)$.

We propose that some tumors elongate their telomeres using TA as opposed to ALT $(6,26,29)$. ALT is a TL maintenance mechanism based on recombination, where telomeres use other telomeric DNA as a template for DNA synthesis. ALT cells are able to elongate their telomeres without any apparent intervention of other telomeres (30). In vitro experiments indicate that human cells may be capable of concomitantly utilizing both mechanisms of telomere maintenance without affecting growth and viability (31).

In summary, we observed that tumors that do not change their TLs have higher TRF1 protein levels than those that do change their TLs. We found a relationship between TL and TRF1 protein abundance, but not with TA. Tumor samples exhibited different molecular activities related to TRF1, TL, and TA when compared with normal samples. These results affirm that a direct relation exists between TL and TRF1 levels. Normal samples with elongated telomeres exhibited higher TRF1 protein levels than samples with short telomeres, a result coincident with the homeostasis telomere hypothesis, whereas tumor samples whose TLs were maintained presented higher TRF1 level. We believe that additional studies of TRF1 will bring to light additional key factors of cancer development. Our study concludes that TRF1 is an important factor in the regulation of the telomeres and TRF1 changes its behavior in cancer compared in normal samples. Then it maybe an important prognostic factor in the future together with telomere length and telomerase activity.

The alteration of the mechanism of TL maintenance (telomerase, TRF1, ALT) may represent a first step toward the proliferation of cells leading to carcinogenesis. TRF1 is an important factor in the homeostasis of telomeres; it is involved in both the telomerase and ALT mechanisms, since TRF1 and TRF2 are required to form ALT structures. TRF1, TL, and TA may therefore serve as new indicators of prognosis and as markers for new anti-cancer therapies.

\section{ACKNOWLEDGMENTS}

The authors thank Ana Martinez (Lleida University) for her help and cooperation in the laboratory.

The present work has been supported by grants from the Fondo de Investigaciones Sanitarias (Health Research Fund) (01/0620) and FEDER. Cristina Valls Bautista is supported by a predoctoral grant from the Generalitat de Catalunya. 


\section{REFERENCES}

1. Blackburn EH. Structure and function of telomeres. Nature 1991; 350:569-73.

2. De Lange T, Shiue L, Myers RM, Cox DR, Naylor SL, Killery AM, et al. Structure and variability of human chromosome ends. Mol Cell Biol 1990;10:518-27.

3. Autexier C, Greider, CW. Telomerase and cancer: revisiting the telomere hypothesis. Trends Biochem Sci 1996;21:387-91.

4. Shay JW, Zou Y, Hiyama E, Wright WE. Telomerase and cancer. Hum Mol Gen 2001;1:677-85.

5. Hiyama E, Hiyama K. Telomerase as tumor marker. Cancer Lett 2003; 194:221-33.

6. Reddel RR. Alternative lengthening of telomeres, telomerase and cancer. Cancer Lett 2003;194:155-62.

7. Saini N, Srinivasan R, Chawala Y, Sharma S, Chakraborti A, Rajwanshi A. Telomerase activity, telomere length and human telomerase reverse transcriptase expression in hepatocellular carcinoma is independent of hepatitis virus status. Liver Int 2009;29:1162-70.

8. Deng Y, Chang S. Role of telomeres and telomerase in genomic instability, senescence and cancer. Lab Invest 2007;87:1071-6.

9. Hug N, Lingner J. Telomere length homeostasis. Chromosoma 2006; 115:413-25.

10. Van Steensel B, de Lange T. Control of telomere length by the human telomeric protein TRF1. Nature 1997;385:740-3.

11. Smogorzewska A, de Lange, T. Regulation of telomerase by telomeric proteins. Annu Rev Biochem 2004;73:177-208.

12. Smogorzewska A, van Steensel B, Bianchi A, Oelmann S, Schaefer MR, Schnapp G, et al. Control of human telomere length by TRF1 and TRF2. Mol Cell Biol 2001;20:1659-68.

13. Garcia-Aranda C, de Juan C, Diaz-Lopez, A, Sanchez-Pernaute A, Torres AJ, Diaz-Rubio E, et al. Correlations of telomere length, telomerase activity and telomeric-repeat binding factor 1 expression in colorectal carcinoma. Cancer 2006;106:541-51.

14. Engelhardt M, Drullinsky P, Guillem J. Telomerase and telomere length in the development and progression of premalignant lesions to colorectal cancer. Clin Cancer Res 1997:122;1141-5.

15. Fujiwara M, Kamma H, Wu W, Yano Y, Homma S, Satoh H. Alternative lengthening of telomeres in the human adrenocortical carcinoma cell line H295R. Int J Oncol 2006;29:445-51.

16. Piatyszek MA, Kim NW, Weinrich SL, Hiyama K, Hiyama E, Woodring EW, et al. Detection of telomerase activity in human cells and tumors by a telomeric repeat amplification protocol (TRAP). Methods Cell Sci 1995;17:1-15.
17. Bautista CV, Felis CP, Espinet JM, García JB, Salas JV. Telomerase activity is a prognostic factor for recurrence and survival in rectal cancer. Dis Colon Rectum 2007;50:611-20.

18. Valls C, Piñol C, Reñé JM, Buenestado J, Viñas J. Telomere length is a prognostic factor for overall survival in colorectal cancer. Colorectal Dis 2011;13:1265-72.

19. Valls Bautista C, Piñol Felis C, Reñe Espinet JM, Buenestado García J, Viñas Salas J. Telomerase activity and telomere length in the colorectal polyp-carcinoma sequence. Rev Esp Enferm Dig 2009; 101(3):179-82.

20. Hsu CP, Miaw J, Hsia JY, Shai SE, Chen CY. Concordant expression of the telomerase-associated genes in non-small cell lung cancer. Eur J Surg Oncol 2003;29:594-9.

21. Colgin LM, Wilkinson C, Englezou A, Kilian A, Robinson MO, Reddel RR. The hTERTalpha splice variant is a dominant negative inhibitor of telomerase activity. Neoplasia 2000;2:426-32.

22. Aragona M, Buda CA, Panetta S, Morelli M, Guiudice A, Campagna FL, et al. Immunohistochemical telomeric-repeat binding factor-1 expression in gastrointestinal tumors. Oncol Rep 2000;7:987-90.

23. Miyachi K, Fujita M, Tanaka N, Sasaki K, Sunagawa M. Correlation between telomerase activity and telomeric-repeat binding factors in gastric cancer. J Exp Clin Cancer Res 2002;21:269-75.

24. Saeed M, Berlin RM, Cruz TD. Exploring the utility of genetic markers for predicting biological age. Leg Med (Tokyo) 2012;14:279-85.

25. Nawrot TS, Straessen JA, Gardenr JP, Aviv A. Telomere length and possible link to X chromosome. Lancet 2004;363:507-10.

26. Hsu CP, Ko JL, Shai SE, Lee LW. Modulation of telomere shelterin by TRF1 (corrected) and TRF2 interacts with telomerase to maintain the telomere length in non-small cell lung cancer. Lung Cancer 2007;58:310-6.

27. La Torre D, de Divitiis O, Conti A, Angileri FF, Cardali S, Aguennouz $\mathrm{M}$, et al. Expression of telomeric repeat binding factor-1 in astroglial brain tumors. Neurosurgery 2005;56:802-10.

28. Cesare AJ, Reddel RR. Alternative lengthening of telomeres: models, mechanisms and implications. Nat Rev Genet 2010;11:319-30.

29. Bollmann FM. Targeting ALT: the role of alternative lengthening of telomeres in pathogenesis and prevention cancer. Cancer Treat Rev 2007;33:704-9.

30. Muntani A, Neumann AA, Hills M, Reddel RR. Telomere elongation involves intra-molecular DNA replication in cells utilizing alternative lengthening of telomeres. Hum Mol Genet 2009;18:1017-27.

31. Cerone MA, Londono-Vallejo JA, Bacchetti, S. Telomere maintenance by telomerase and by recombination can coexist in human cells. Hum Mol Genet 2001;18:1945-52. 